\title{
Skutterudites, a thermoelectric material investigated by high field Mössbauer spectroscopy
}

\author{
M. Reissner • E. Bauer • W. Steiner • P. Rogl • \\ A. Leithe-Jasper · Yu. Grin
}

Published online: 10 October 2008

(C) The Author(s) 2008. This article is published with open access at Springerlink.com

\begin{abstract}
The partly filled skutterudites $\mathrm{A}_{x} \mathrm{Fe}_{4} \mathrm{Sb}_{12}(\mathrm{~A}=\mathrm{La}, \mathrm{Pr}, \mathrm{Nd}, \mathrm{Eu}, \mathrm{Yb}$, and the monovalent homologue $\mathrm{Tl}$ ) were investigated at $4.2 \mathrm{~K}$ by ${ }^{57} \mathrm{Fe}$ Mössbauer spectroscopy in external fields up to $13.5 \mathrm{~T}$. Two Fe sites were identified. The observed relative spectral areas are not for all compounds in agreement with filling factors for the A-atoms determined from X-ray experiments. The change in sign and the small value of the induced hyperfine fields for magnetically ordered compounds are strong hints for itinerant ferromagnetism with small ordered moments.
\end{abstract}

Keywords Skutterudites • Itinerant magnetism • High field Mössbauer spectroscopy

\section{Introduction}

The search for improved thermoelectric materials renewed the interest in the, at least partly, filled ternary skutterudites $\mathrm{A}_{x} \mathrm{M}_{4} \mathrm{X}_{12}$, where $\mathrm{M}$ denotes one of the transition metals $\mathrm{Co}, \mathrm{Fe}, \mathrm{Ru}$ or $\mathrm{Os}, \mathrm{X}$ a pnictogen $(\mathrm{P}, \mathrm{As}$, or $\mathrm{Sb}$ ), and $\mathrm{A}$ represents the filler atoms in the large empty voids present in this cubic structure (Im $\overline{3}$ ). Exchanging $\mathrm{P}$ by $\mathrm{Sb}$ offers the possibility to increase interatomic distances without changing crystal structure. The number of charge carriers can be widely tuned since the filler atoms A span from alkali- over alkaline-earth metals, rare earths to actinides. These combinations generate such a diverse behavior as unconventional superconductivity,

M. Reissner $(\varangle) \cdot$ E. Bauer · W. Steiner

Institut für Festkörperphysik, Technische Universität Wien, Vienna, Austria

e-mail: reissner@ifp.tuwien.ac.at

P. Rogl

Institut für Physikalische Chemie, Universität Wien, Vienna, Austria

A. Leithe-Jasper · Yu. Grin

Max-Planck-Institut für Physik fester Stoffe, Dresden, Germany 
magnetism, and large thermoelectric effects [see e.g. 1-4]. The latter reveal the possibility to tailor the thermoelectric figure of merit $Z T=(\sigma / \kappa) S^{2} T(\sigma$ is the electrical conductivity, $\kappa$ the electronic and lattice contribution to the thermal conductivity, and $S$ the Seebeck coefficient), which for state of the art materials should be in the order of one.

In spite of the large amount of theoretical and experimental investigations performed on these compounds, precise knowledge about low temperature Femoments is still missing [e.g. [4] and references herein]. To obtain a more detailed knowledge about the magnetic ground state, we performed ${ }^{57} \mathrm{Fe}$ high field Mössbauer measurements on a large variety of Fe based compounds and like to focus in the present report on those built up with $\mathrm{Sb}$ and $\mathrm{A}=\mathrm{La}, \mathrm{Pr}, \mathrm{Nd}, \mathrm{Eu}, \mathrm{Yb}$, and $\mathrm{Tl}$.

$\mathrm{LaFe}_{4} \mathrm{Sb}_{12}$ is nonmagnetic, at least down to $2 \mathrm{~K}$, with $\mu_{\text {eff }}=3.0 \mu_{\mathrm{B}} / \mathrm{fu}$ derived from susceptibility measurements at higher temperatures [5]. Because $\mathrm{La}^{3+}$ possess no magnetic moment, the measured one has to be attributed to the $\left(\mathrm{Fe}_{4} \mathrm{Sb}_{12}\right)$-building block. Band structure calculations indicate hybridization of the La states with $\mathrm{Sb}$ and Fe states resulting in an enhanced effective mass for the two highest occupied bands [6]. Furthermore a double peak structure of the 3d-DOS in the proximity of the Fermi energy was obtained, from which the presence of a moment on the Fe atoms was concluded [7]. Recent studies reveal the importance of spin fluctuations and a behavior close to a ferromagnetic quantum critical point [8].

The Pr compound orders magnetically around $5 \mathrm{~K}$ with presumably some kind of antiferromagnetic spin alignment on the Pr ions and an effective moment of 2.7 $\mu_{\mathrm{B}}$ on the $\left(\mathrm{Fe}_{4} \mathrm{Sb}_{12}\right)$ building blocks, if a moment according to $\mathrm{Pr}^{3+}$ is assumed. As ground state for the Pr atoms the triplet $\Gamma_{5}$ was deduced $[5,9,10]$. Interestingly, upon increasing the filling fraction to almost $100 \%$ a singlet ground state and no magnetic ordering is observed [11].

The $\mathrm{Nd}$ and Eu compounds order magnetically at $13 \mathrm{~K}$ and around $84 \mathrm{~K}$, with $\mu_{\text {eff }}=4.5$ and $8.4 \mu_{\mathrm{B}} / \mathrm{fu}$, respectively [5]. The presence of a mixture of $\mathrm{Eu}^{2+}$ and $\mathrm{Eu}^{3+}$ in $\mathrm{EuFe}_{4} \mathrm{Sb}_{12}$ was proposed recently [12]. In case of $\mathrm{YbFe}_{4} \mathrm{Sb}_{12}$ it has been shown [13] that $\mathrm{Yb}$ is stable divalent and the effective moment of $\mu_{\mathrm{eff}}=4.49 \mu_{\mathrm{B}} / \mathrm{fu}$ originally attributed to intermediate valency [14] is solely due to itinerant paramagnetism of the $\left(\mathrm{Fe}_{4} \mathrm{Sb}_{12}\right)$ building blocks. Nevertheless, high-resolution photoemission studies points to small $4 \mathrm{f}^{13}$ contributions depending on penetration depth, which can be explained by surface oxidation effects [15].

Similar to the alkali-metal compounds formed with $\mathrm{Na}$ and $\mathrm{K}$ [16], $\mathrm{Tl}$ is also monovalent. Magnetic order is observed below $80 \mathrm{~K}$ and spin polarized LDA calculations give a ferromagnetic ground state with moments on Fe of $0.82 \mu_{\mathrm{B}}$ [17].

\section{Experimental}

Details of sample preparation were described in [16-18]. The filling factor for the A atoms was determined from XRD data, by using FULLPROF [19] and in the case of the second Eu sample by hot neutron measurements.

Mössbauer measurements were carried out in transmission geometry at $4.2 \mathrm{~K}$ in external fields $\left(B_{\mathrm{a}}\right)$ up to $13.5 \mathrm{~T}$ (accuracy $\pm 0.01 \mathrm{~T}$, homogeneity $0.1 \%$ ). The field direction was always parallel to the $\gamma$-ray direction. The ${ }^{57} \mathrm{CoRh}$ source (relative to which all center shift (CS) values are given) was situated in a field-compensated 
region $(<0.1 \mathrm{~T})$. The source temperature varies in the course of the measurements between 5 and $25 \mathrm{~K}$.

All spectra were analysed by solving the full Hamiltonian taking into account both electrostatic and magnetic hyperfine interactions. Sample thickness was considered using the approximation given by Morup and Both [20]. The spectra recorded in external fields were analyzed with the restriction that the relative area of the subspectra, center shift, and quadrupole splitting, QS, remain constant and only the measured hyperfine field, $B_{\mathrm{hf}}$, changes due to the presence of the external field. Deviations of the line width up to $10 \%$ were allowed.

\section{Results and discussion}

For the ${ }^{57} \mathrm{Fe}$-probe atom the nearest neighbor shell is formed by Sb octahedra, and the next nearest by twelve Sb (with slightly different distances) and two A atoms (Fig. 1). From X-ray investigations both the Fe and the $\mathrm{Sb}$ sublattice are found to be fully occupied, whereas considerable amounts of vacancies are present in the Asublattice ( $x=0.80,0.73,0.72$, and 0.88 for $\mathrm{La}, \mathrm{Pr}, \mathrm{Nd}$, and $\mathrm{Eu}$ (Eu1), respectively). For $\mathrm{Yb}$ the A sublattice appears to be fully occupied [14]. Furthermore, a second Eu sample (Eu2) with $x=0.95$ and a Tl compound with a high degree of filling $(x=0.982)$ were investigated [17]. Although crystallographically only one Fe site is present in the structure, at least two subspectra (with the exception of $\mathrm{Yb}$ ) were necessary to analyze both the zero field and the in-field spectra in a consistent way (Fig. 2). The line widths were always in the order of $0.22 \mathrm{~mm} / \mathrm{s}$.

If voids are present, and if they are statistically distributed in the corresponding neighbor shell, the ${ }^{57} \mathrm{Fe}$-probe atom may be surrounded by two, one, or zero A atoms. These different surroundings can influence the hyperfine parameters leading to the asymmetry in the recorded spectra. The relative intensities of the resulting subspectra are in fair agreement with the void concentration determined by the $\mathrm{X}$-ray investigations for $\mathrm{A}=\mathrm{La}, \mathrm{Pr}, \mathrm{Nd}, \mathrm{Yb}$ and Eu1. This agreement led to the allocation of the subspectrum with the high area ratio to Fe completely surrounded by $\mathrm{A}$ atoms and of the subspectrum with the low area ratio to Fe surrounded by one or zero A atoms (resolution of the latter is not possible within the statistical accuracy of the present investigations). A similar scenario was suggested for the Co-based skutterudites $\mathrm{Tl}_{0.8} \mathrm{Co}_{3} \mathrm{FeSb}_{12}$ and $\mathrm{Tl}_{0.5} \mathrm{Co}_{3.5} \mathrm{Fe}_{0.5} \mathrm{Sb}_{12}$ by Long et al. [21], but called into question, because of deviations of observed area ratio of the subspectra from the ones following from a statistical distribution.

For Eu the situation gets more complicated if mixed valent behavior, as proposed by Krishnamurthy et al. [12] is taken into consideration. A proper mixture of $\mathrm{Eu}^{3+}$ and $\mathrm{Eu}^{2+}$ could be responsible for the low total moment observed by magnetic measurements [22] and for the appearance of different subspectra. But to explain magnetic and Mössbauer results for all samples in a consistent way the degree of mixture of the two valence states must depend on void concentration. Experiments, which confirm this assumption, are presently missing.

For the $\mathrm{Tl}$ compound a void concentration of at least $10 \%$ follows from the area ratio of the subspectra of approx. 80:20 taking into account a binomial distribution. This is, however, much too high according to the results obtained for the filling factor from chemical and X-ray analyses. This discrepancy, which is also present in our 
Fig. 1 Fe environments in $\mathrm{AFe}_{4} \mathrm{Sb}_{12}$. Only the nearest $\mathrm{Sb}$ shell is shown

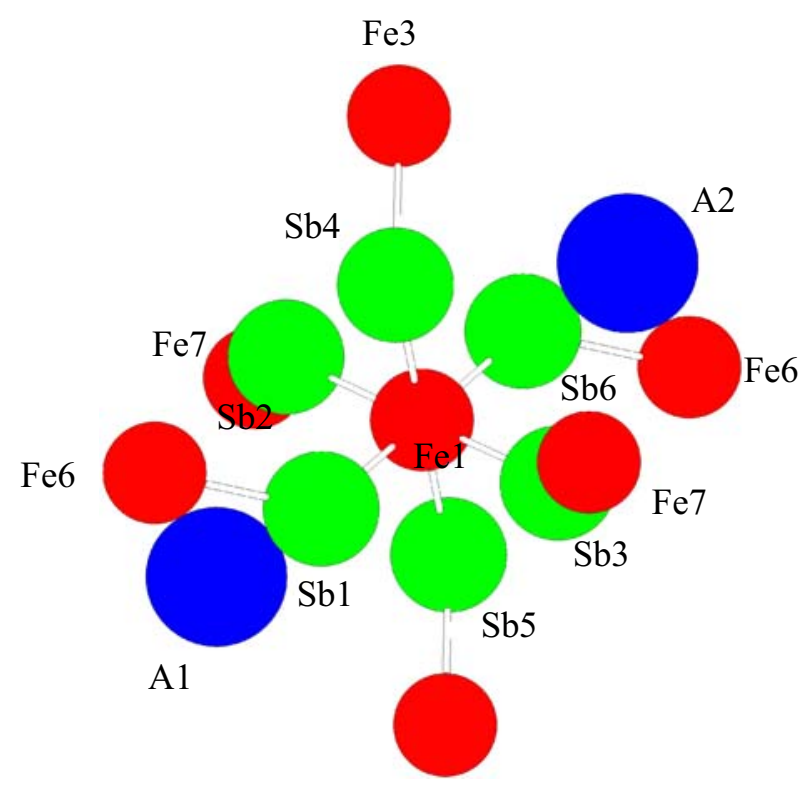

$\mathrm{Fe} 3$

investigations of other alkali and alkaline-earth samples, makes the interpretation of the two different Fe sites in terms of voids in the A shell difficult. However, with the exception of $\mathrm{TlCo}_{3} \mathrm{FeSb}_{12}$ [23] at present no experimental clue or theoretical hints exist for other interpretations of the difference in charge density at the Fe site in metallic skutterudites. For sub-stoichiometric Co-based skutterudites the existence of $\mathrm{CoSb}_{3}$ offers the possibility of another approach. A solid solution of a completely filled $\mathrm{Fe}$ compound in an unfilled Co compound was assumed to be realized in $\mathrm{Ce}_{x} \mathrm{Fe}_{4-y} \mathrm{Co}_{y} \mathrm{Sb}_{12}[24,25]$.

The obtained QS values for the various compounds scatter around $0.25 \mathrm{~mm} / \mathrm{s}$ and are different for the two Fe positions. For both sites the QS values for the (most probably) mixed valent Eu skutterudites (e.g. at $4.2 \mathrm{~K}: 0.170$ and $0.180 \mathrm{~mm} / \mathrm{s}$ for Fe with large and small area ratio, respectively) are approximately $44 \%$ to $50 \%$ smaller than those for the $\mathrm{RE}^{3+}$ compounds (e.g. $\mathrm{Pr}_{0.73} \mathrm{Fe}_{4} \mathrm{Sb}_{12}$ at $4.2 \mathrm{~K}: 0.245$ and $0.270 \mathrm{~mm} / \mathrm{s}$, allocation as above) and are in rough agreement with the ones obtained for $\mathrm{Tl}^{1+}$ (e.g. $\mathrm{Tl}_{0.98} \mathrm{Fe}_{4} \mathrm{Sb}_{12}$ at $4.2 \mathrm{~K}: 0.170$ and $0.175 \mathrm{~mm} / \mathrm{s}$, allocation as above). The lattice parameters at room temperature are $9.1369 \AA$ for Pr, $9.1631 \AA$ for Eu, and $9.1973 \AA$ for Tl. The field gradient at the Fe nucleus reflects the hybridisation of the Fe-d and Sb-p states which seems to be influenced by the appearing changes in the interatomic distances but less by the charge carrier densities resulting from the filler atoms. This is in accordance with the observed small temperature dependence of QS in $\mathrm{Eu}$ and $\mathrm{Tl}$ skutterudites.

Good agreement exists of the CS values found in the present investigation with the one reported for the Ce compounds (in performing the proper correction for the source temperature) [24]. CS values for the monovalent $\mathrm{Tl}$ at $4.2 \mathrm{~K}$ are 0.22 and $0.33 \mathrm{~mm} / \mathrm{s}$ (allocation as above). This indicates a rather similar s-electron density at the Fe nucleus independent of the valency of the filler atoms as long as the 

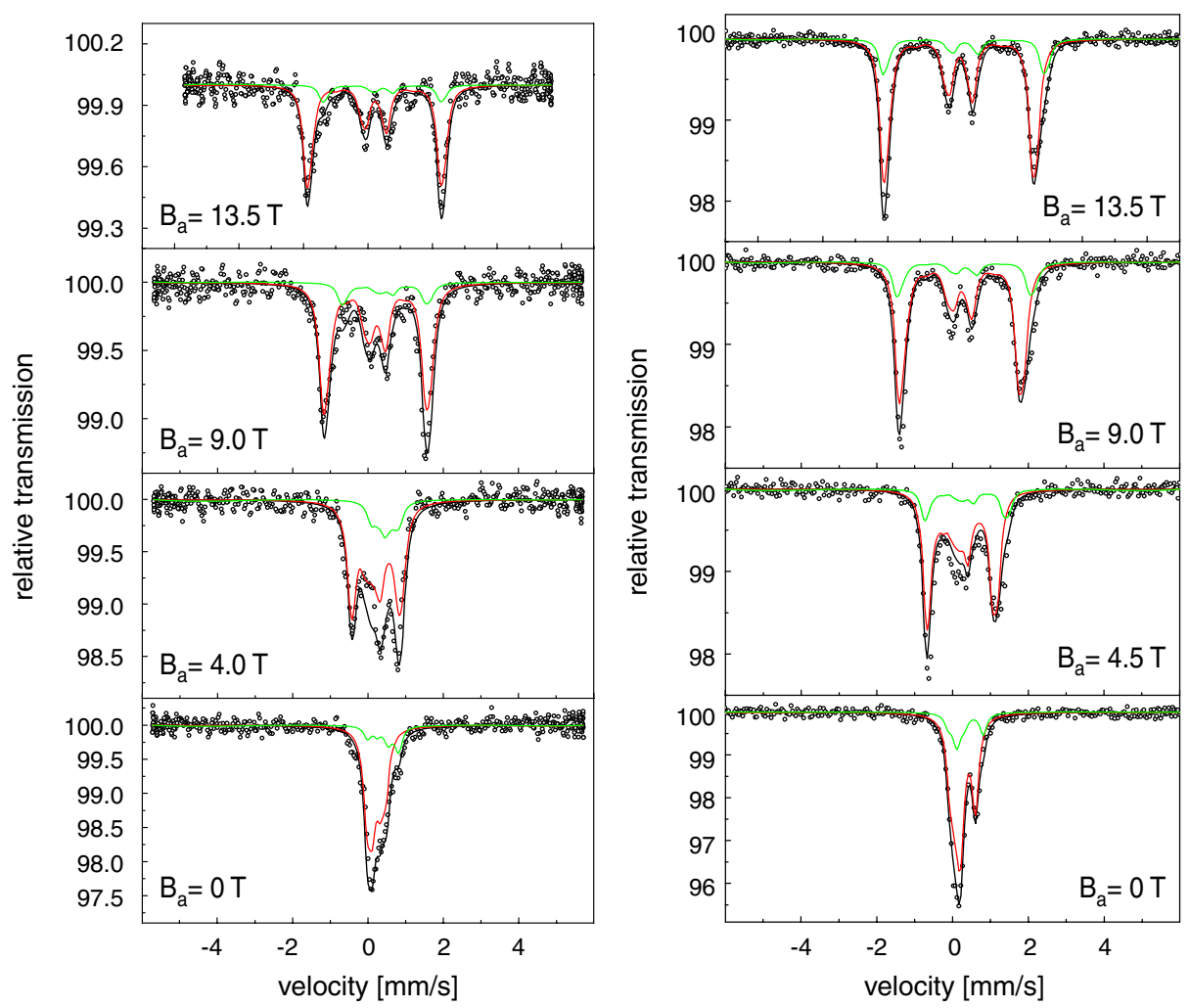

Fig. 2 Typical spectra recorded at $4.2 \mathrm{~K}$ in various external magnetic fields for $\mathrm{Eu}_{0.88} \mathrm{Fe}_{4} \mathrm{Sb}_{12}$ (left) and $\mathrm{Tl}_{0.98} \mathrm{Fe}_{4} \mathrm{Sb}_{12}$ (right)

interatomic distances are comparable (lattice parameter $a$ remains in the order of 9.1 $\AA$ ). The presence of a high spin $\mathrm{Fe}^{2+}$ state can be excluded.

Above $4 \mathrm{~T}$ the spectra are fully polarized (the intensity of the $\Delta m=0$ transitions vanishes, e.g. Fig. 2). For compounds without magnetic order [La and $\mathrm{Yb} 5,14]$ the values of the measured hyperfine fields for the subspectrum allocated to the component with the large area either coincide with the one of $B_{\text {a }}$ or were slightly larger. Significant deviations from the value of $B_{\text {a }}$ were only obtained for Fe atoms allocated to the spectra with the small area. Similar behavior is obtained for the $\mathrm{Pr}$ and $\mathrm{Nd}$ compounds, although according to bulk magnetic measurements they are magnetically ordered at the measuring temperature (ordering temperatures 5 and $13 \mathrm{~K}$ for $\mathrm{Pr}[9]$ and Nd [5], respectively). The Eu and Tl compounds are magnetically ordered, up to about $84 \mathrm{~K}[5,18]$ and $80 \mathrm{~K}$ [17], respectively. From $B_{\text {ind }}=B_{\text {hf }}-B_{\text {a }}$ the induced hyperfine fields were calculated. In the $\mathrm{Pr}$ and $\mathrm{Nd}$ compounds for $\mathrm{Fe}$ atoms allocated to the smaller spectral area $B_{\text {ind }}$ exhibits some tendency towards saturation at high applied fields, whereas for Fe allocated to the large area $B_{\text {ind }}$ scatters around zero (Fig. 3). The change in sign of $B_{\text {ind }}$ obtained for $\mathrm{Eu}$ and $\mathrm{Tl}$ compounds (appr. same ordering temperature) indicates that valence and core contributions to the hyperfine field are of comparable magnitude. The opposite sign of these contributions allows a compensation which may explain the small values 


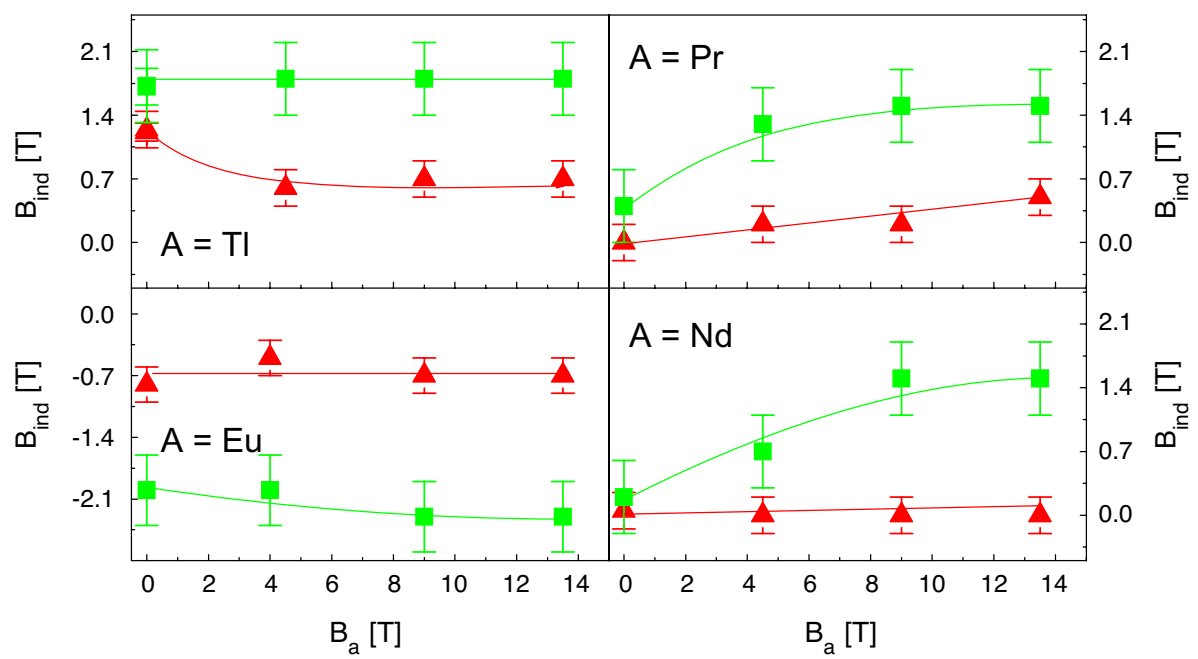

Fig. 3 Field dependence of the induced hyperfine fields $B_{\text {ind }}$ at $4.2 \mathrm{~K}$ for magnetically ordered $\mathrm{Tl}_{0.98} \mathrm{Fe}_{4} \mathrm{Sb}_{12}, \mathrm{Eu}_{0.88} \mathrm{Fe}_{4} \mathrm{Sb}_{12}, \mathrm{Pr}_{0.73} \mathrm{Fe}_{4} \mathrm{Sb}_{12}$, and $\mathrm{Nd}_{0.72} \mathrm{Fe}_{4} \mathrm{Sb}_{12}$ (triangle large, square small spectral area). Lines are only guides for the eyes

of $B_{\text {ind. }}$. This is strongly supported by ASW band structure calculations (Blaha and Mohn, private communication). Only the core-field reflects the spin density at the ${ }^{57} \mathrm{Fe}$ nucleus and is thus directly proportional to the Fe moment. This contribution is not directly accessible in the present experiments. However, the change in sign and the numerical value observed for $B_{\text {ind }}$ indicates that this contribution might be too small to explain directly the large effective moment assigned to the $\left(\mathrm{Fe}_{4} \mathrm{Sb}_{12}\right)$ building blocks from bulk magnetic measurements at higher temperatures. It contradicts the simple picture based on charge counting arguments that the large moments on the $\left(\mathrm{Fe}_{4} \mathrm{Sb}_{12}\right)$ building blocks are due to the unpaired spin of $\mathrm{Fe}^{3+}$ in low spin configuration [5] and point to itinerant ferromagnetism with small ordered moments as proposed for the alkali-metal $\mathrm{Na}, \mathrm{K}$, and the homologue Tl skutterudites.

\section{Conclusions}

The asymmetry in the intensity of the outer lines of the spectra recorded by infield measurements was accounted for by fits with two subspectra. The observed relative spectral areas are in fair agreement with filling factors determined from $\mathrm{X}$ ray experiments for $\mathrm{A}=\mathrm{La}, \mathrm{Pr}, \mathrm{Nd}$, and $\mathrm{Yb}$, however fail for the other compounds. At present no consistent explanation for the allocation of the second component is possible.

The values of the induced hyperfine fields are much too small to explain directly the large effective moments deduced on the Fe sites from susceptibility measurements at high temperatures. The change in sign and the small value of $B_{\text {ind }}$ obtained at $4.2 \mathrm{~K}$ for magnetically ordered compounds point to itinerant ferromagnetism with small ordered moments. 
Acknowledgements Work supported by the EU-NoE "Complex Metallic Alloys" and Austrian FWF project P13778.

Open Access This article is distributed under the terms of the Creative Commons Attribution Noncommercial License which permits any noncommercial use, distribution, and reproduction in any medium, provided the original author(s) and source are credited.

\section{References}

1. Sales, B.C.: Filled skutterudites. In: Gschneidner, K.A., Jr., Buenzli, J.C.G., Pecharsky, V.K. (eds.) Handbook on the Physics and Chemistry of Rare Earths, vol. 33, pp. 1-34. Elsevier Science, Amsterdam (2003)

2. Cichorek, T., Mota, A.C., Steglich, F., Frederick, N.A., Yuhasz, W.M., Maple, M.B.: Pronounced enhancement of the lower critical field and critical current deep in the superconducting state of $\mathrm{PrOs}_{4} \mathrm{Sb}_{12}$. Phys. Rev. Lett. 94(107002), 1-4 (2005)

3. Maple, M.B., Bauer, E.D., Frederick, N.A., Ho, P.C., Yuhasz, W.M., Zapf, V.S.: Strongly correlated electron phenomena in filled skutterudite compounds. Physica B 328, 29-33 (2003)

4. Uher, C.: Skutterudites prospective novel thermoelectrics. In: Tritt, T.M. (ed.) Recent Trends in Thermoelectric Materials Research I, Semiconductors and Semimetals, vol. 69, pp. 139-253. Academic, New York (2001)

5. Danebrock, M.E., Evers, C.B., Jeitschko, W.: Magnetic properties of alkaline earth and lanthanoid iron antimonides $\mathrm{AFe}_{4} \mathrm{Sb}_{12}(\mathrm{~A}=\mathrm{Ca}, \mathrm{Sr}, \mathrm{Ba}, \mathrm{La}-\mathrm{Nd}, \mathrm{Sm}, \mathrm{Eu})$ with the $\mathrm{LaFe}_{4} \mathrm{P}_{12}$ structure. J. Phys. Chem. Solids 57, 381-387 (1996)

6. Singh, D.J., Mazin, I.I.: Calculated thermoelectric properties of La-filled skutterudites. Phys. Rev. B. 56, R1650-R1653 (1997)

7. Takegahara, K., Harima, H.: Electronic band structure of filled skutterudite $\mathrm{YbFe}_{4} \mathrm{P}_{12}$. J. Phys. Soc. Jpn. 71(Suppl.), 240-242 (2002)

8. Viennois, R., Charar, S., Ravot, D., Haen, P., Mauger, A., Bentien, A., Paschen, S., Steglich, F.: Spin fluctuations in the skutterudite compound $\mathrm{LaFe}_{4} \mathrm{Sb}_{12}$. Eur. Phys. J. B 46, 257-267 (2005)

9. Bauer, E., Berger, S., Paul, Ch., Della Mea, M., Hilscher, G., Michor, H., Reissner, M., Steiner, W., Grytsiv, A., Rogl, P., Scheidt, E.W.: Crystal field effects and thermoelectric properties of $\mathrm{LaFe}_{4} \mathrm{P}_{12}$. Phys. Rev. B. 66(214421), 1-9 (2002)

10. Reissner, M., Bauer, E., Steiner, W., Rogl, P.: High field Mössbauer and magnetic investigations of $\mathrm{Pr}_{0.73} \mathrm{Fe}_{4} \mathrm{P}_{12}$. J. Magn. Magn. Mat. 272-276, 813-815 (2004)

11. Tanaka, K., Kawahito, Y., Yonezawa, Y., Kikuchi, D., Aoki, H., Kuwahara, K., Ichihara, M., Sugawara, H., Aoki, Y., Sato, H.: Nonmagnetic ground state in fully filled $\operatorname{Pr}_{x} \mathrm{Fe}_{4} \mathrm{Sb}_{12}(\mathrm{x}=1.0)$ synthezised under high pressure. J. Phys. Soc. Jpn. 76(103704), 1-4 (2007)

12. Krishnamurthy, V.V., Lang, J.C., Haskel, D., Keavney, D.J., Srajer, G., Robertson, J.L., Sales, B.C., Mandrus, D.G., Singh, D.J., Bilc, D.I.: Ferrimagnetism in $\mathrm{EuFe}_{4} \mathrm{Sb}_{12}$ due to the interplay of f-electron moment and a nearly ferromagnetic host. Phys. Rev. Lett. 98(126403), 1-4 (2007)

13. Schnelle, W., Leithe-Jasper, A., Schmidt, M., Rosner, H., Borrmann, H., Burkhardt, U., Mydosh, J.A., Grin, Yu.: Itinerant iron magnetism in filled skutterudites $\mathrm{CaFe}_{4} \mathrm{Sb}_{12}$ and $\mathrm{YbFe}_{4} \mathrm{Sb}_{12}$ : stable divalent state of ytterbium. Phys. Rev. B 72(020402(R)), 1-4 (2005)

14. Leithe-Jasper, A., Kaczorowski, D., Rogl, P., Bogner, J., Reissner, M., Steiner, W., Wiesinger, G., Godart, C.: Synthesis, crystal-structure determination and physical properties of $\mathrm{YbFe}_{4} \mathrm{Sb}_{12}$. Solid State Commun. 109, 395-400 (1999)

15. Dedkov, Y.S., Molodtsov, S.L., Rosner, H., Leithe-Jasper, A., Schnelle, W., Schmidt, M., Grin, Yu.: Divalent state of ytterbium in $\mathrm{YbFe}_{4} \mathrm{Sb}_{12}$ filled skutterudites. Physica C 460-462, 698-699 (2007)

16. Leithe-Jasper, A., Schnelle, W., Rosner, H., Baenitz, M., Rabis, A., Gippius, A.A., Morozova, E.N., Borrmann, H., Burkhardt, U., Ramlau, R., Schwarz, U.M., Mydosh, J.A., Grin, Yu., Ksenofontov, V., Reiman, S.: Weak itinerant ferromagnetism and electronic and crystal structures of alkaline-metal iron antimonides: $\mathrm{NaFe}_{4} \mathrm{Sb}_{12}$ and $\mathrm{KFe}_{4} \mathrm{Sb}_{12}$. Phys. Rev. B 70(214418), 1-12 (2004)

17. Leithe-Jasper, A., Schnelle, W., Rosner, H., Cardoso-Gil, R., Baenitz, M., Mydosh, J.A., Grin, Yu., Reissner, M., Steiner, W.: $\mathrm{TlFe}_{4} \mathrm{Sb}_{12}$ : Weak itinerant ferromagnetic analogoue to alkalimetal iron antimony skutterudites. Phys. Rev. B 77(064412), 1-9 (2008) 
18. Bauer, E., Berger, S., Galatanu, A., Galli, M., Michor, H., Hilscher, G., Paul, Ch., Ni, B., Abd-Elmeguid, M.M., Tran, V.H., Grytsiv, A., Rogl, P.: Crystal structure and physical properties of $\mathrm{Eu}_{0.83} \mathrm{Fe}_{4} \mathrm{Sb}_{12}$. Phys. Rev. B 63(224414), 1-7 (2001)

19. Rodriguez-Carjaval, J.: Recent advances in magnetic structure determination by neutron powder diffraction. Physica B 192, 55-69 (1993)

20. Morup, S., Both, E.: Interpretation of Mössbauer spectra with broadened lines. Nucl. Instr. Meth. 124, 435-8 (1975)

21. Long, G.J., Mathieu, B., Sales, B.C., Hermann, R.P., Grandjean, F.: Electronic structure of thallium filled skutterudites studied by x-ray absorption and Mössbauer spectroscopy. J. Appl. Phys. 92, 7236-7241 (2002)

22. Reissner, M., Bauer, E., Steiner, W., Hilscher, G., Grytsiv, A., Rogl, P.: $\mathrm{Eu}_{z} \mathrm{Fe}_{4} \mathrm{Sb}_{12}$ studied by ${ }^{57} \mathrm{Fe}$ Mössbauer spectroscopy. Physica B 378-380, 232-234 (2006)

23. Ghosez, P., Veithen, M.: First principles study of filled and unfilled antimony skutterudites. J. Phys., Condens. Matter 19(096002), 1-19 (2007)

24. Long, G.J., Hautot, D., Grandjean, F., Morelli, D.T., Meissner, G.P.: Mössbauer effect study of filled antimonide skutterudites. Phys. Rev. B 60, 7410-7418 (1999)

25. Meissner, G.P., Morelli, D.T., Hu, S., Yang, J., Uher, C.: Structure and lattice thermal conductivity of fractionally filled skutterudites: solid solutions of fully filled and unfilled end members. Phys. Rev. Lett. 80, 3551-3554 (1998) 